\title{
Perceptual adaptation to facial asymmetries
}

\author{
Gillian Rhodes, Kim Louw, And Emma Evangelista \\ University of Western Australia, Crawley, Australia
}

\begin{abstract}
Humans and other animals are highly sensitive to deviations from bilateral symmetry and prefer symmetric mates. Fluctuating asymmetries (FAs) are random deviations from perfect symmetry that can result from developmental instability. Human perceptions of facial asymmetry are driven by FAs and insensitive to directional asymmetries (DAs), which have a consistent direction of bias (e.g., left side always larger) across the population and are unrelated to developmental stability. We hypothesized that perceptual adaptation may filter out DAs and provide a proximate mechanism for this perceptual focus on FAs. We created a small population of faces with DAs by applying a unilateral distortion to the same side of each face. After 5 min of adaptation, (new) faces with these DAs looked less asymmetric and the most symmetric-looking distortion shifted toward the adapting asymmetry level. Parallel changes occurred for attractiveness. We suggest that perceptual adaptation may provide the proximate mechanism for an evolutionarily adaptive focus on FAs.
\end{abstract}

Humans and many other animals are highly sensitive to deviations from bilateral symmetry, and they prefer symmetric mates (Møller \& Swaddle, 1997; Rhodes, 2006; Rhodes \& Simmons, 2007). This preference may be an evolutionary adaptation for finding high-quality mates, given the link between developmental instability and fluctuating asymmetry (FA; Livshits \& Kobyliansky, 1991; Mather, 1953; Møller \& Thornhill, 1998; Polak, 2003; Van Valen, 1962; Zakharov, 1981). FA is characterized by random deviations from perfect symmetry in bilaterally paired traits (e.g., left eye equally likely to be larger or smaller than right eye) that arise when environmental stress and/or genetic factors disrupt development (Møller \& Swaddle, 1997; Polak, 2003). In contrast, directional asymmetry (DA) has a consistent bias across a population (e.g., left eye always larger than right eye) and does not reflect developmental instability (Polak, 2003).

Interestingly, human perceptions of facial symmetry are driven largely by FA and are unrelated to DA (Simmons, Rhodes, Peters, \& Koehler, 2004). ${ }^{1}$ Given that humans find symmetric faces attractive (for reviews, see Rhodes, 2006; Rhodes \& Simmons, 2007), this focus on facial FA could be evolutionarily adaptive if facial FA covaries negatively with mate quality. Evidence that facial asymmetry is elevated in some chromosomal disorders (Thornhill \& Møller, 1997) suggests that it might. In addition, facial asymmetry has been linked with poor health in nonclinical populations, although this finding remains controversial (for a recent review, see Rhodes \& Simmons, 2007).

What proximate mechanism could filter out DA and focus perception on asymmetries that may signal developmental instability? A good candidate is perceptual adaptation, which occurs when response properties change in response to changes in the inputs (Clifford \& Rhodes, 2005;
Clifford et al., 2007; Schwartz, Hsu, \& Dayan, 2007). Perceptual adaptation may serve to calibrate responses to the distribution of inputs experienced and would ensure sensitivity to distributional properties of the inputs, such as whether an asymmetric trait in a face is FA or DA.

Numerous studies have reported perceptual adaptation in face perception (MacLin \& Webster, 2001; Rhodes, Jeffery, Watson, Clifford, \& Nakayama, 2003; Rhodes et al., 2004; Robbins, McKone, \& Edwards, 2007; Watson \& Clifford, 2003; Webster \& MacLin, 1999). In these studies, participants typically view a series of faces to which a consistent distortion has been applied. For example, the internal features may have been "expanded" spherically from a central point, making the faces look as if they were viewed through a fish-eye lens. After the viewer has adapted to these faces, the distortion becomes less noticeable and faces with slightly expanded features look more normal than undistorted faces.

We hypothesized that perceptual adaptation to consistent, directional asymmetries in a population of faces could explain the observed perceptual focus on facial FA (Simmons et al., 2004). Two studies have investigated perceptual adaptation to facial asymmetry: Morikawa (2005) found that after participants adapted to asymmetric eye height in a single face, this face looked more normal. However, he did not examine generalization to new faces. Robbins et al. (2007) adapted their participants to asymmetric eyes in two known identities and asked them to adjust the eye positions in those faces and in those of two other known identities until the faces looked most like the original faces. Adaptation occurred in both cases, suggesting that it might be possible to adapt to consistent asymmetries in a population of faces. However, the effect was extremely small (a mean of one pixel or less). Moreover,

G. Rhodes, gill@psy.uwa.edu.au 


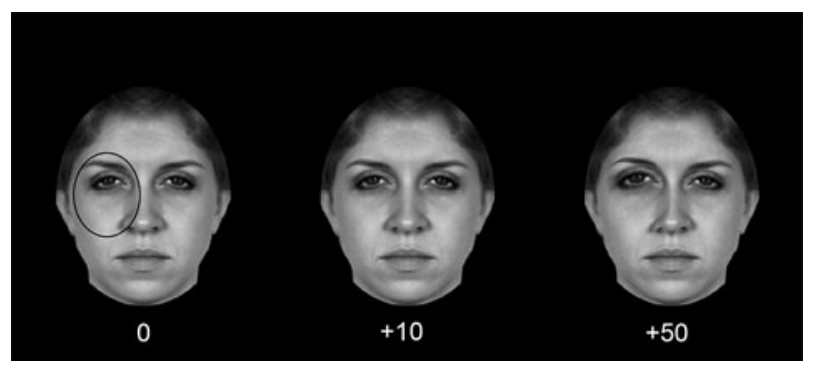

Figure 1. A perfectly symmetric face ( 0 , left) made by blending an undistorted face with its mirror image. Unilateral expansion distortions were applied to a region on one side of the face (see oval) to produce adapting faces with small $(+10$, center) and large (+50, right) asymmetric distortions. These distortions expand the selected region about a central point, enlarging the eye and shifting it upward and making the side of the nose thinner.

neither study examined effects of adaptation on perceived symmetry or preferences.

In the present study, we tested whether people adapt to DAs in a population of unfamiliar faces, so that those asymmetries become perceptually less salient. Participants adapted to 10 faces with consistent asymmetric distortions made by expanding an oval-shaped region on either the left or right side of each face (see Figure 1). These unilateral distortions were applied to perfectly symmetric versions of the adapting faces, so that only asymmetries produced by these distortions were present. Before and after adapting, participants rated the symmetry of test faces taken from face continua displaying a range of unilateral distortion levels, from expansion distortions, through no distortion, to contraction distortions (Figure 2). The side of distortion always matched that of the adapting faces. These test continua are analogous to those used to measure adaptation to bilateral distortions (Rhodes et al., 2003), except that the distortions are unilateral. The adapting and test faces were always different identities. We examined adaptation to small as well as large asymmetries (between participants), to see whether adaptation would occur for both subtle and obvious facial asymmetries. We predicted that, after adaptation, the adapting asymmetry would look more symmetric and that the most symmetric-looking distortion would shift away from perfect symmetry toward the adapting distortion.
We also predicted that adaptation to consistent asymmetries would increase the attractiveness of those asymmetries. This prediction was motivated by both the appeal of facial symmetry (for reviews, see Rhodes, 2006; Rhodes \& Simmons, 2007) and evidence that adaptation to distortions increases the attractiveness of those distortions (Rhodes et al., 2003). To ensure independent assessment, different participants rated attractiveness and symmetry. Parallel effects of adaptation on attractiveness and symmetry judgments would implicate perceptual adaptation in face preferences and in symmetry perception.

\section{METHOD}

\section{Participants}

Participants were young adults (17-26 years old $)^{2}$ recruited from the University of Western Australia. Initially, we recruited 24 symmetry raters and 24 attractiveness raters. However, noisy data meant that curves could not be fitted (see below) for 13 attractiveness raters (either poor fits, defined as $R^{2}<.60$, or no clear maximum), and an additional 33 raters were required to obtain a final sample of 24 attractiveness raters. The final groups consisted of 19 female and 5 male symmetry raters and 16 female and 8 male attractiveness raters.

\section{Stimuli}

Twenty ( 10 female) perfectly symmetric, grayscale, full-face photographs of adults with neutral expressions were taken from Rhodes, Proffit, Grady, and Sumich (1998). These face images were made perfectly symmetric by blending original photographs with their mirror images (see Rhodes et al., 1998, for details). Ten faces were used to create adapting stimuli, and 10 were used to create test stimuli.

To create the adapting stimuli, we applied asymmetric distortions to each face, using the spherize distortion function in Adobe Photoshop. Small $(+10)$ and large $(+50)$ expansion distortions were applied to an oval-shaped area on the left side of each face (Figure 1) in a region that included the eye and the side of the nose but excluded the mouth (to avoid generating expressions by raising or lowering one side of the mouth). The distortions expand the selected region about a central point, making the left side of the nose thinner and enlarging the left eye and shifting it upward. The resulting images were mirror reversed about the vertical axis of symmetry to create a second set of asymmetric adapting stimuli with distortions on the right side of the face.

For each of the 10 test faces, we created an 11-face distortion continuum (see Figure 2) that included contraction distortions $(-50$, $-40,-30,-20,-10)$, no distortions ( 0 ), and expansion distortions $(+10,+20,+30,+40,+50)$. The distortions were applied to the same oval-shaped area on the left of the face as was used for the

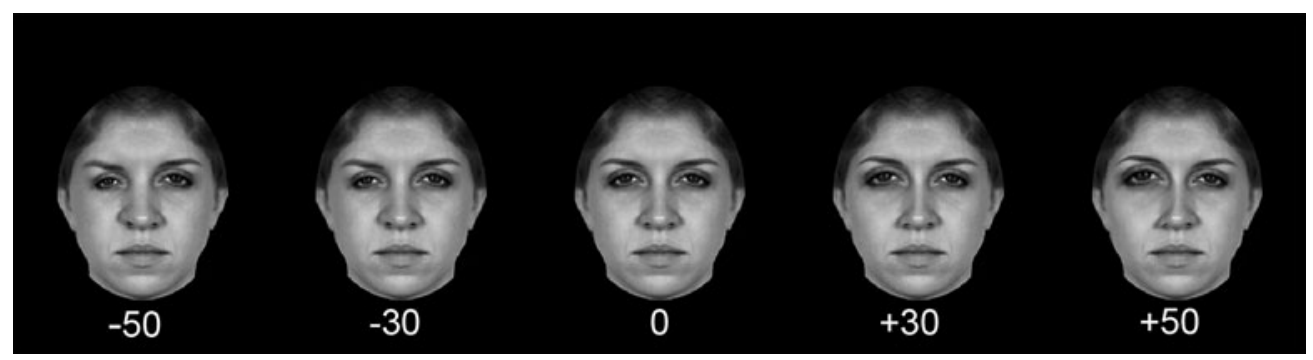

Figure 2. Selected images from a test continuum. Test continua contained 11 versions of the same face, with unilateral distortions varying from strongly contracted $(-50)$ through no distortion $(0$, perfect symmetry) to strongly expanded (+50). Contraction distortions have the opposite effect of expansion distortions, making the side of the nose thicker and reducing the size of the eye and shifting it down. All distortions were applied to the same side of the face. Left-sided distortions are shown here. 
adapting faces. Mirror images were created to generate a second test set with right-sided distortions. All images were shown in black oval masks that hid the outer hairline but not the inner hairline or face outline. Images measured $8.6^{\circ} \times 11.3^{\circ}(217 \times 285$ pixels, shown at 72 pixels per inch) and were viewed from a distance of $50 \mathrm{~cm}$.

\section{Procedure}

The session began with a preadaptation rating phase, in which all 110 test face images ( 10 faces $\times 11$ distortion levels) were presented in a random sequence and were rated for either symmetry or attractiveness (between participants) on a 1-9 scale $(1=$ asymmetric, $9=$ symmetric $; 1=$ unattractive, $9=$ attractive $)$. Each face appeared for $1,500 \mathrm{msec}$, with the word "RATE" above and below it, followed by the rating scale (attractiveness or symmetry). The rating scale remained on the screen until a response was made, which initiated the next trial. Participants were encouraged to use the full range of the scale. There were three practice trials.

Next came a 5-min adaptation phase, during which 10 asymmetrically distorted adapting faces were shown repeatedly in a random sequence, each appearing for $4 \mathrm{sec}$ with a 200 -msec interstimulus interval (ISI). The distortions were unilateral and always on the same side, so that the direction of asymmetry was consistent. The distortions could be either weak (+10 expanded) or strong $(+50$ expanded). Side and strength of distortion side were counterbalanced across participants. Participants were instructed to attend to each of the images.

The postadaptation rating phase followed. This was the same as the preadaptation rating phase, except that each test image was preceded by a randomly selected adapting face $(8 \mathrm{sec})$ and a 500 -msec blank ISI.

\section{RESULTS}

\section{Symmetry}

Mean symmetry ratings, averaged across participants, were plotted as a function of test distortion level, before and after participants had adapted to small $(+10)$ and large $(+50)$ asymmetric distortions. Before adaptation, the most symmetric-looking faces were close to perfect symmetry, but after adaptation, choices were biased toward the adapting asymmetry (Figure 3 ). This shift in perceived symmetry was most evident for large adapting distortions but was also present for small adapting distortions. Following Rhodes et al. (2003), we fitted third-order polynomials to each function for each participant. Their maxima were used to estimate the test distortion levels that looked most symmetric, before and after adapting (Figure 4). Fits were good (mean $R^{2}=.87, S D=.08$, minimum $R^{2}=$ $.67 ; 1$ participant was excluded due to poor fit, $R^{2}<.60$ ). Another participant, whose fitted curves had no clear maxima, was also excluded.

Distortions chosen as most symmetric. We conducted a three-way ANOVA on the mean distortion level chosen as most symmetric, with size (small, large) and side (left, right) of adapting asymmetry as betweensubjects factors and test time (preadaptation, postadaptation) as a repeated measures factor. Planned $t$ tests were used for follow-up comparisons. Inspection of Figure 4 shows that the most symmetric-looking distortion shifted in the direction of the adapting asymmetry, with a larger shift for the larger adapting distortion. This was confirmed by a significant interaction between test time and size of adapting asymmetry $\left[F(1,18)=4.79, p<.05, \eta_{\mathrm{p}}^{2}=.21\right]$. Both shifts were significant [small, $t(18)=2.40$,

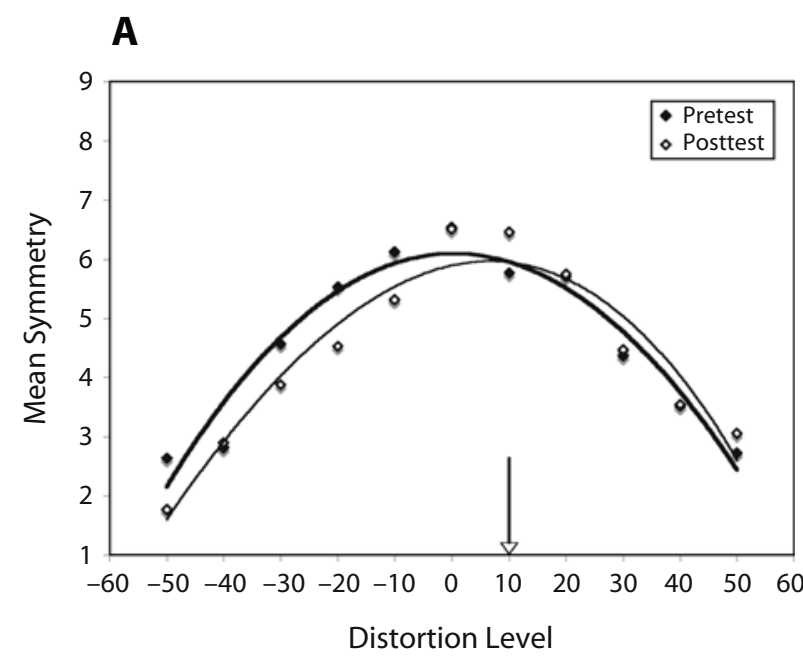

B

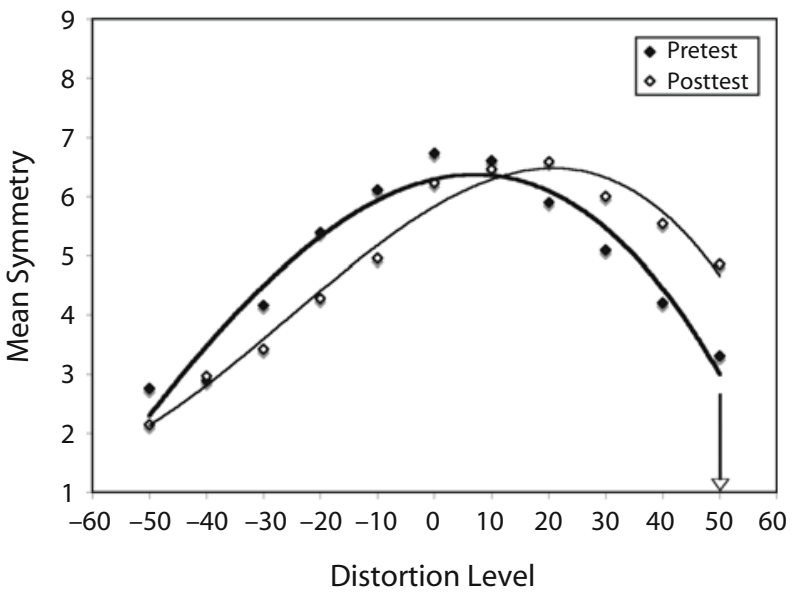

Figure 3. Mean symmetry ratings as a function of distortion level, before (pretest) and after (posttest) adaptation to (A) small $\left(+10\right.$ : pretest, $R^{2}=.93$; posttest, $\left.R^{2}=.93\right)$ and large $(+50$ : pretest, $R^{2}=.94$; posttest, $R^{2}=.98$ ) asymmetric distortions. Arrows indicate adapting asymmetry levels.

$p<.03$; large, $t(18)=5.51, p<.0001]$. After adaptation, choices were significantly biased away from perfect symmetry $(0)$ for both small $[M=5.9, S E=2.0 ; t(18)=$ $2.92, p<.01]$ and large $[M=20.0, S E=2.0 ; t(18)=$ $9.82, p<.001]$ adapting distortions. Before adapting, the most symmetric distortion $(M=4.1, S E=1.2)$ differed slightly from perfect symmetry $[t(18)=3.42, p<.01]$, but inspection of Figure 3 suggests that this may be an artifact of large expanded (positive) asymmetries looking more symmetric than large contracted (negative) asymmetries did.

There were significant main effects of test time (preadaptation, $M=4.1, S E=1.2$; postadaptation, $M=13.0$, $S E=1.4)\left[F(1,18)=31.08, p<.0001, \eta_{\mathrm{p}}^{2}=.63\right]$ and size of adapting asymmetry (small, $M=3.2, S E=1.5$; large, $M=13.8, S E=1.5)[F(1,18)=24.91, p<.0001$, $\left.\eta_{\mathrm{p}}^{2}=.58\right]$. No other effects were significant (all $F \mathrm{~s}<2.52$ $p \mathrm{~s}>.12$ ). 


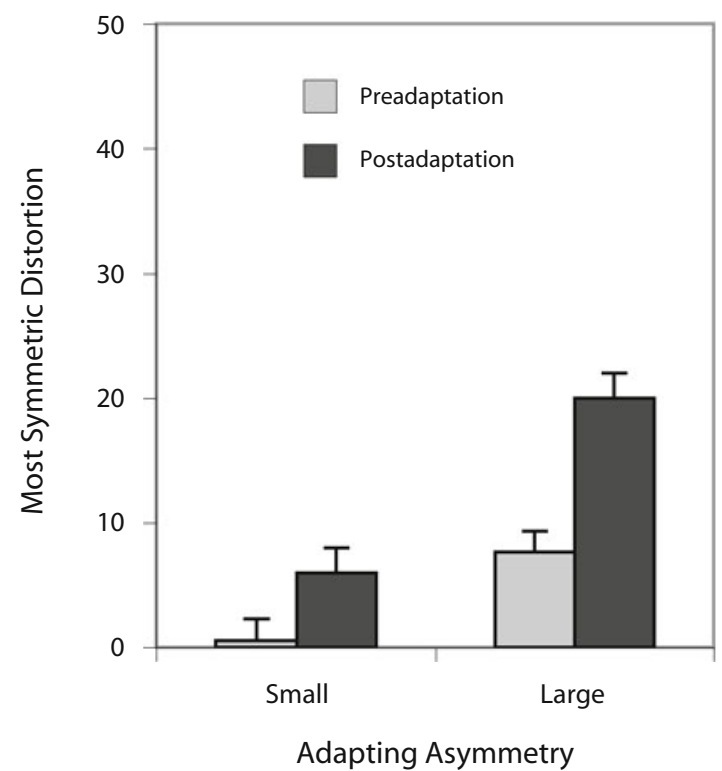

Figure 4. Most symmetric-looking distortion level before and after adapting to small $(+10)$ and large $(+50)$ asymmetric distortions. Standard error bars are shown.

Perceived symmetry of adapting asymmetry levels. A second ANOVA was conducted on the mean symmetry ratings for the adapting asymmetry levels $(+10$ or +50$)$. These asymmetries, indicated by arrows in Figure 3, became less noticeable after adaptation, particularly for large asymmetries. Specifically, the adapting asymmetries looked more symmetric after adapting $(M=5.7, S E=$ $0.2)$ than before $(M=4.5, S E=0.2)[F(1,18)=28.92$, $\left.p<.0001, \eta_{\mathrm{p}}^{2}=.62\right]$, and test time interacted with size of adapting asymmetry $\left[F(1,18)=5.58, p<.03, \eta_{\mathrm{p}}^{2}=\right.$ .24]. The increase was significant for small (before, $M=$ 5.8, $S E=0.3$; after, $M=6.5, S E=0.3)[t(18)=2.14$, $p<.05]$ and large (before, $M=3.1, S E=0.3$; after, $M=4.8, S E=0.3)[t(18)=5.50, p<.0001]$ adapting asymmetries.

Not surprisingly, faces with small asymmetries were rated as more symmetric $(M=6.2, S E=0.3)$ than were those with large asymmetries $(M=4.0, S E=0.3)$ $\left[F(1,18)=36.96, p<.0001, \eta_{\mathrm{p}}^{2}=.67\right]$. The only other significant effect was an interaction between size and side of adapting asymmetry $\left[F(1,18)=12.64, p<.002, \eta_{\mathrm{p}}^{2}=\right.$ .41]. The difference in symmetry ratings between faces with small and large asymmetries was larger when the asymmetric distortions were on the right (small, $M=6.8$, $S E=0.4$; large $M=3.3, S E=0.3$ ) than when they were on the left (small, $M=5.5, S E=0.3$; large, $M=4.6$, $S E=0.4$ ) sides of the faces. This interaction has no clear theoretical significance and is not discussed further.

\section{Attractiveness}

Mean attractiveness ratings were plotted against test distortion level, before and after adapting, for each participant. Polynomials were fitted to these curves in order to estimate the test distortion levels that looked most at- tractive before and after adapting (mean $R^{2}=.85, S D=$ .09 , minimum $R^{2}=.61$ ). Figure 5 shows the averaged data for 24 raters with acceptable curve fits. As predicted, the most attractive distortion shifted toward the adapting asymmetry, at least for large adapting distortions. This was confirmed by the same analyses as for symmetry ratings. Two participants were excluded from these analyses because their fitted curves had no clear maxima.

Distortions chosen as most attractive. The preferred distortion shifted toward the adapting asymmetry level, particularly for large adapting asymmetries (Figures 5 and 6). This was confirmed by a significant effect of test time $\left[F(1,18)=26.42, p<.0001, \eta_{\mathrm{p}}^{2}=.60\right]$ and a significant interaction with size of adapting asymmetry $\left[F(1,18)=7.99, p<.02, \eta_{\mathrm{p}}^{2}=.31\right]$. Planned tests confirmed that the shift was significant for large adapting asymmetries $[t(18)=5.45, p<.0001]$ and marginally significant for small adapting asymmetries $[t(18)=1.73$,

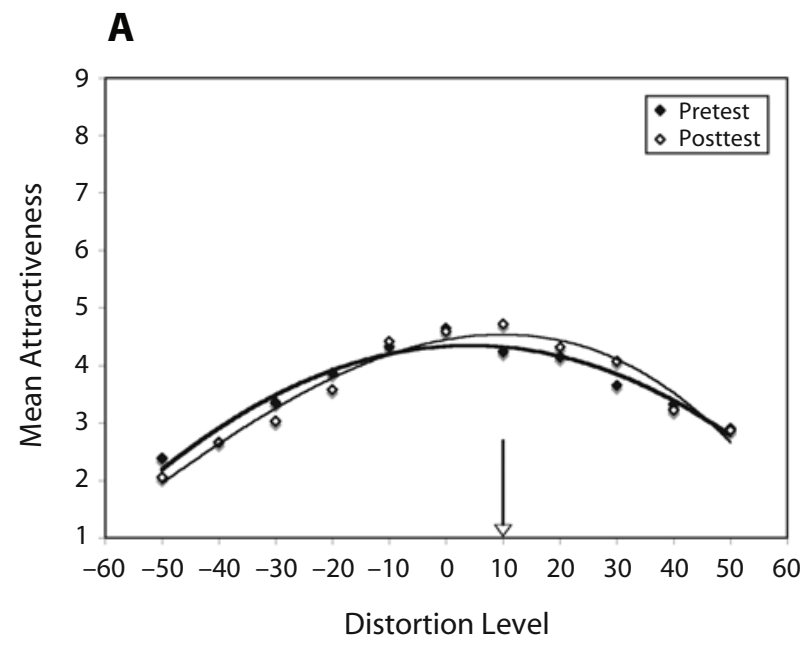

\section{B}

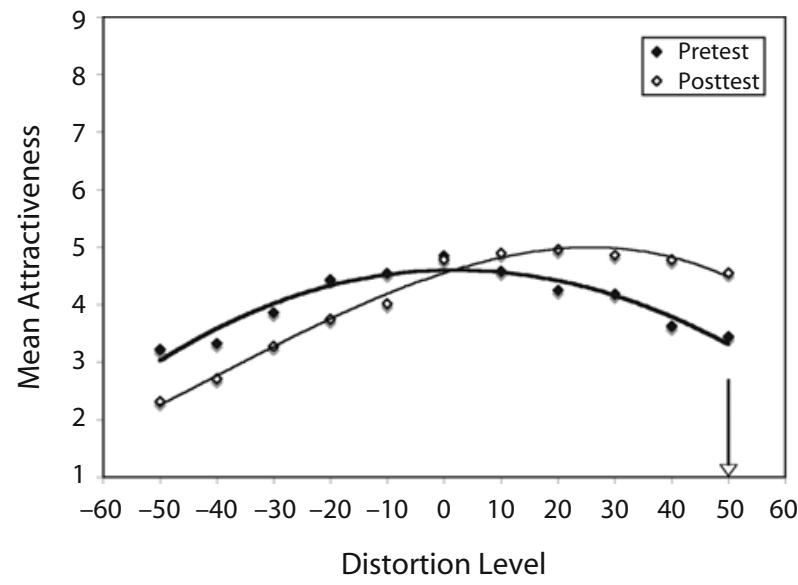

Figure 5. Mean attractiveness ratings as a function of distortion level, before (pretest) and after (posttest) adaptation to (A) small $\left(+10\right.$ : pretest, $R^{2}=.95$; posttest, $\left.R^{2}=.95\right)$ and $(B)$ large $(+50$ : pretest, $R^{2}=.95$; posttest, $R^{2}=.99$ ) asymmetric distortions. Arrows indicate adapting asymmetry levels. 


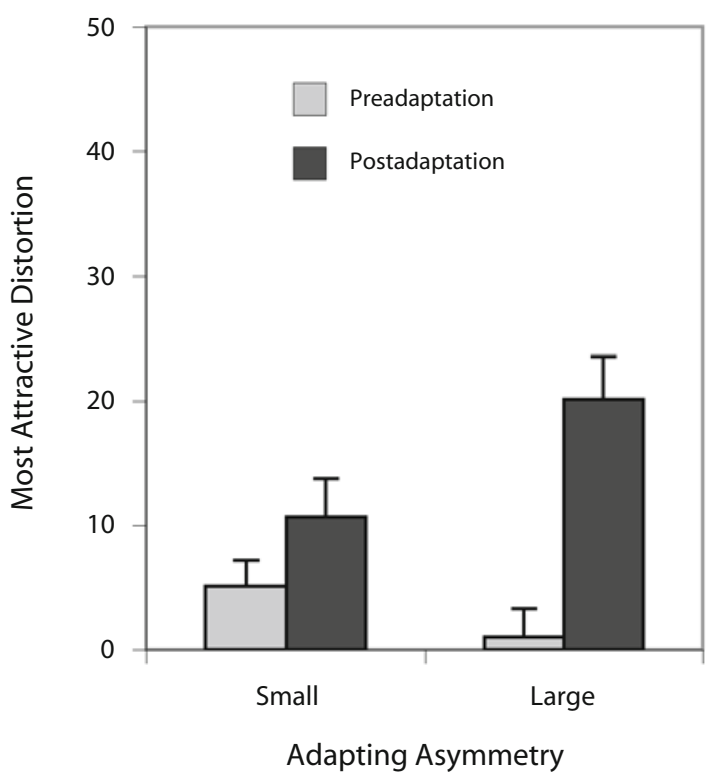

Figure 6. Most attractive distortion level before and after adapting to small $(+10)$ and large $(+50)$ asymmetric distortions. Standard error bars are shown.

$p<.10]$. No other effects were significant. Before adapting, the preferred distortion $(M=3.0, S E=1.5)$ did not differ significantly from perfect symmetry $(0)[t(18)=$ 1.98 , n.s.]. In sum, when curves could be fitted to attractiveness data, adaptation to asymmetry produced shifts in face preferences comparable to those observed in symmetry perception (cf. Figures 4 and 6).

Attractiveness of adapting asymmetry levels. The adapting asymmetry levels $(+10$ or +50$)$, indicated by arrows in Figure 5, became significantly more attractive after adaptation (after, $M=4.6, S E=0.2$; before, $M=$ $3.8, S E=0.2)\left[F(1,18)=23.20, p<.0001, \eta_{\mathrm{p}}^{2}=.56\right]$. No other effects were significant, although there was a marginal interaction between test time and size of adapting asymmetry $\left[F(1,18)=4.11, p<.06, \eta_{\mathrm{p}}^{2}=.19\right]$, with a larger increase in attractiveness for larger distortions. Not surprisingly, more asymmetric faces $(+50)$ were rated as less attractive $(M=3.9, S E=0.2)$ than were less asymmetric ones $(+10, M=4.5, S E=0.2)$, although this effect was only marginally significant $[F(1,18)=3.12, p<$ $.10]$. To confirm that these results were not an artifact of excluding many participants ( 33 out of 57 excluded), ${ }^{3}$ we repeated this analysis using the original sample of 24 participants. Again, the adapting asymmetry levels became more attractive after adapting $(M=4.4, S E=0.2$, after; $M=4.0, S E=0.2$, before) $[F(1,20)=4.55, p<.05$, $\left.\eta_{\mathrm{p}}^{2}=.19\right]$, and small adapting asymmetries were significantly more attractive $(M=4.7, S E=0.3)$ than large ones $(M=3.7, S E=0.3)\left[F(1,20)=7.17, p<.02, \eta_{\mathrm{p}}^{2}=.26\right]$. No other effects were significant. These results confirm that adaptation to DAs increases their appeal.

\section{Comparison of Symmetry and Attractiveness}

We conducted a MANOVA with the most symmetric and most attractive distortion levels as two dependent variables (DVs), size and side of adapting asymmetry as between-participant factors, and test time as a repeated measures factor. The only interaction involving type of DV was with size $\left[F(1,36)=4.48, p<.05, \eta_{\mathrm{p}}^{2}=.11\right]$, where the difference between small and large adapting asymmetries was greater for the symmetry (small, $M=$ 3.2, $S E=1.8$; large, $M=13.8, S E=1.8)$ than the attractiveness (small, $M=7.9, S E=1.8$; large, $M=10.6$, $S E=2.0)$ DV. Importantly, the type of DV did not interact with test time $(F<1)$, suggesting that adaptation to asymmetric distortions has parallel effects on perceptions of symmetry and attractiveness.

\section{DISCUSSION}

Adapting to DAs in a small population of faces reduced sensitivity to those asymmetries and made faces with low levels of those asymmetries appear symmetric. These results suggest that perceptual adaptation could provide a proximate mechanism for the evolutionarily adaptive perceptual focus on FA in human faces (Simmons et al., 2004).

Adaptation effects were observed after only a few minutes of exposure, and larger effects would be expected with ongoing exposure to naturally occurring facial DAs, which, by definition, appear repeatedly in a population of faces. Adaptation was also observed for very subtle $(+10 \%)$ as well as for more obvious $(+50 \%)$ asymmetries. Therefore, although the degree of asymmetry present in individual faces may be small (Simmons et al., 2004), adaptation seems likely. Certainly, people adapt readily to the asymmetries present in an individual face so that its mirror image appears asymmetric (Paras, Kaping, \& Webster, 2004). Our results suggest that they also rapidly adapt to DAs in a population of faces.

We observed larger perceptual shifts for more extreme adapting asymmetries. In contrast, Robbins et al. (2007) observed smaller shifts after adapting to more extreme asymmetries in vertical eye position. Their statistical effect was driven by the most extreme asymmetry level, which was anatomically impossible and may not have fully engaged face processing mechanisms. Norm-based and multichannel models of face coding make different predictions about the expected relationship between size of adapting distortion and the resulting perceptual shifts (Robbins et al., 2007). The present results are consistent with a norm-based coding model in which face dimensions are coded by pairs of neural populations tuned to belowand above-average values (Rhodes \& Jeffery, 2006).

Adaptation to asymmetries affected preferences as well as perceived symmetry, with the most attractive asymmetry level shifting away from perfect symmetry toward the adapting distortion. This preference shift mirrors the shift in what looks most symmetric and is consistent with a preference for symmetric faces (Rhodes, 2006; Rhodes \& Simmons, 2007). ${ }^{4}$ We were unable to estimate optimal attractiveness levels for about half of our participants, who did not appear to find symmetry attractive under the present conditions (with noisy and/or flattish functions). Therefore, caution is needed in interpreting the attractive- 
ness results. However, a similar picture emerges if we simply consider the attractiveness of the adapting asymmetry levels, which increased after exposure for all participants. We conclude, therefore, that parallel effects of adaptation occurred for symmetry perception and attractiveness.

Our results show that the visual system is sensitive to the distribution of asymmetries in a population of faces, with perceptual adaptation reducing the perceptual salience of those asymmetries that have a consistent directional bias (i.e., of DAs). Perceptual adaptation may also reduce the salience of DA in other morphological traits (e.g., body traits), allowing symmetry perception and preferences to focus on FA. To the extent that FA signals mate quality, this focus would be evolutionarily adaptive. We conclude that perceptual adaptation could provide a proximate mechanism for an evolutionarily adaptive perceptual focus on FAs.

\section{AUTHOR NOTE}

This research was funded by a professorial fellowship from the Australian Research Council to G.R. We thank Leigh Simmons for helpful discussions about this work and Hanne Lie, Louise Ewing, and Linda Jeffery for comments on the manuscript. Address correspondence to G. Rhodes, School of Psychology, University of Western Australia, 35 Stirling Highway, Crawley, WA 6009, Australia (e-mail: gill@psy.uwa.edu.au).

\section{REFERENCES}

Clifford, C. W. G., \& Rhodes, G. (Eds.) (2005). Fitting the mind to the world: Adaptation and after-effects in high-level vision. Oxford: Oxford University Press.

Clifford, C. W. G., Webster, M. A., Stanley, G. B., Stocker, A. A., Kohn, A., Sharpee, T. O., \& Schwartz, O. (2007). Visual adaptation: Neural, psychological and computational aspects. Vision Research, 47, 3125-3131.

Livshits, G., \& KobYliansky, E. (1991). Fluctuating asymmetry as a possible measure of developmental homeostasis in humans: A review. Human Biology, 63, 441-466.

MacLin, O. H., \& Webster, M. A. (2001). Influence of adaptation on the perception of distortions in natural images. Journal of Electronic Imaging, 10, 100-109.

Mather, K. (1953). Genetic control of stability in development. Heredity, 7, 297-336.

Møller, A. P., \& Swaddle, J. P. (1997). Asymmetry, developmental stability, and evolution. New York: Oxford University Press.

MøLler, A. P., \& Thornhill, R. (1998). Bilateral symmetry and sexual selection: A meta-analysis. American Naturalist, 151, 174-192.

MoriKawA, K. (2005). Adaptation to asymmetrically distorted faces and its lack of effect on mirror images. Vision Research, 45, 3180-3188.

Palmer, A. R., \& Strobeck, C. (2003). Fluctuating asymmetry analyses revisited. In M. Polak (Ed.), Developmental instability: Causes and consequences (pp. 279-319). New York: Oxford University Press.

Paras, C. L., Kaping, D., \& Webster, M. A. (2004). Adaptation and the perception of facial symmetry [Abstract]. Journal of Vision, 4(8), 440a. doi:10.1167/4.8.440
PolaK, M. (ED.) (2003). Developmental instability: Causes and consequences. New York: Oxford University Press.

RHODES, G. (2006). The evolutionary psychology of facial beauty. Annual Review of Psychology, 57, 199-226.

RhoDEs, G., \& JEFFERY, L. (2006). Adaptive norm-based coding of facial identity. Vision Research, 46, 2977-2987.

Rhodes, G., Jeffery, L., Watson, T., Clifford, C. W. G., \& NAKAYAMA, K. (2003). Fitting the mind to the world: Face adaptation and attractiveness aftereffects. Psychological Science, 14, 558-566.

Rhodes, G., Jeffery, L., Watson, T., JaQuet, E., Winkler, C., ClifFORD, C. W. G. (2004). Orientation-contingent face aftereffects and implications for face-coding mechanisms. Current Biology, 14, 2119 2123.

Rhodes, G., Proffit, F., Grady, J. M., \& Sumich, A. (1998). Facial symmetry and the perception of beauty. Psychonomic Bulletin \& Review, 5, 659-669.

Rhodes, G., \& Simmons, L. (2007). Symmetry, attractiveness and sexual selection. In R. I. M. Dunbar \& L. Bartlett (Eds.), Oxford handbook of evolutionary psychology (pp. 333-364). New York: Oxford University Press.

Robbins, R., McKone, E., \& Edwards, M. (2007). Aftereffects for face attributes with different natural variability: Adaptor position effects and neural models. Journal of Experimental Psychology: Human Perception \& Performance, 33, 570-592.

Schwartz, O., Hsu, A., \& DAYAN, P. (2007). Space and time in visual context. Nature Reviews Neuroscience, 8, 522-535.

Simmons, L. W., Rhodes, G., Peters, M., \& Koehler, N. (2004). Are human preferences for facial symmetry focused on signals of developmental instability? Behavioral Ecology, 15, 864-871.

Thornhill, R., \& Møller, A. P. (1997). Developmental stability, disease and medicine. Biological Reviews of the Cambridge Philosophical Society, 72, 497-548.

VAN VAlen, L. (1962). A study of fluctuating asymmetry. Evolution, 16, 125-142.

Watson, T. L., \& Clifford, C. W. G. (2003). Pulling faces: An investigation of the face-distortion aftereffect. Perception, 32, 1109-1116.

Webster, M. A., \& MacLin, O. H. (1999). Figural aftereffects in the perception of faces. Psychonomic Bulletin \& Review, 6, 647-653.

ZAJONC, R. B. (1968). Attitudinal effects of mere exposure. Journal of Personality \& Social Psychology, 9, 1-27.

ZAKHAROV, V. M. (1981). Fluctuating asymmetry as an index of developmental homeostatis. Genetika, 13, 241-256.

\section{NOTES}

1. Symmetry ratings were also affected by traits showing random deviations around a consistent DA, which may also signal developmental instability (Palmer \& Strobeck, 2003).

2. One 48-year-old participant was tested, but her data were excluded because clear maxima could not be found on her curves.

3. Most of the excluded participants had noisy and rather flat functions, suggesting little consistent effect of asymmetry level on their preferences.

4. A similar shift has been observed after adaptation to symmetric facial distortions (Rhodes et al., 2003). Neither is simply a mere exposure effect (Zajonc, 1968), because the adapting and test faces are different identities.

(Manuscript received October 14, 2008; revision accepted for publication December 9, 2008.) 